\title{
Intelligent Transportation System with Diverse Semi-Autonomous Vehicles
}

\author{
Rahul Kala \\ Robotics and Artificial Intelligence Laboratory, \\ Indian Institute of Information Technology, Allahabad, 211012, India \\ E-mail:rkala001@gmail.com \\ www.rkala.in \\ Kevin Warwick \\ Vice Chancellors Office, Coventry University, Priory Street, \\ Coventry, CV1 5FB, Coventry, United Kingdom \\ E-mail:k.warwick@coventry.ac.uk \\ www.kevinwarwick.com
}

Received 28 October 2014

Accepted 9 June 2015

\begin{abstract}
It is becoming increasingly likely that in the future most vehicles will be semi-autonomous with communication capabilities and will possess diverse speed capabilities. The aim of this paper is to describe the creation of an integrated traffic simulation system for such settings. While behaviours such as crossing, overtaking, etc. are trivially exhibited, the implemented system includes an intelligent traffic lights operating system, dynamic speed limits for speed lanes, lane reservation, road reservation, density regularization or traffic jam avoidance, replanning for blockages and re-routing under slow traffic conditions.
\end{abstract}

Keywords: Traffic Simulation, Intelligent Transportation Systems, Semi-Autonomous Vehicles, Vehicle Planning.

\section{Introduction}

For reasons of safety, driving efficiency, and sometimes driving comfort, much research is now being done in the domain of driving assistance systems and autonomous vehicles. Advanced Driver Assistance Systems (ADAS) are aimed at helping the driver by various technological means. Common features of such systems include fatigue alert ${ }^{1}$, collision alert ${ }^{2}$, safety caution alert ${ }^{3}$, overtaking vehicle alert, obstacle detection ${ }^{4}$, route planning and guidance ${ }^{5}$, sign recognition $^{6}$, etc. Autonomous vehicles are technologically more advanced and are capable of driving on their own without any human input, making all driving decisions on their own ${ }^{7,8}$. Semi-autonomous vehicles however provide limited capabilities, restricted to either or all of automated parking ${ }^{9}$, overtaking ${ }^{10}$, lane following, ${ }^{11}$ etc.
State-of-the art research in these domains showcases a promising future in which vehicles increasingly become more advanced, to the extent that most vehicles on the road will be semi-autonomous with communication abilities, access to advanced travel information and dynamic route guidance systems, amongst other safety and decision making systems. In reality though, non-intelligent vehicles may still exist in small numbers for a very long time. A fully autonomous scenario, with only autonomous vehicles is also a possibility, although this may take a much longer time to materialize.

Vehicles with different levels of autonomy have different capabilities related to vision, control and reaction time, which all lead to capabilities to drive at different maximum speeds. Autonomous vehicles may especially be designed for different commercial or 
business requirements due to which they may vary in their type, make and performance, leading to different driving speeds. For semi-autonomous vehicles driving speeds also depend upon driver preference, passenger preference, purpose of travel, social stature, etc. All of which leads to different vehicles driving at different speeds. In fact at present traffic showcases limited diversity in driving speeds, wherein different drivers prefer to drive at different speeds, leading to lane changing, overtaking and a distribution of traffic across lanes roughly as per the preferential driving speeds. Increasing levels of autonomy are though very likely to increase this speed band, making traffic more diverse in terms of speed capabilities.

Inter-vehicle communication ${ }^{12}$ systems help a group of vehicles to talk to each other and share information which provides advantages including collision warning, ${ }^{13}$ obstacle alert, ${ }^{14}$ cooperative obstacle avoidance, ${ }^{15} \quad$ etc. $\quad$ Vehicle-infrastructure communication $^{16}$ meanwhile enables a vehicle to communicate with a transportation infrastructure to be found on the road. Such a system may be useful to communicate traffic or road conditions to the vehicle. Communication helps vehicles to take optimal decisions at the local and global level, while reducing any uncertainties. Having extra information via communication, unknown to the driver's or the vehicle's normal vision, is always helpful in decision making. Here the focus is not only on making a vehicle's personal plan better, but in enabling vehicles to collaborate to make the overall transportation system better, even if it is at the cost of one's own personal plan.

Traffic systems play a major role in regulating the movement of vehicles in any country. In most scenarios static traffic rules lead to reasonable traffic management for most general driving. Traffic may further be managed by making rules changes for certain days and times (e.g. heavy vehicles at night only) or to cope with certain scenarios (e.g. possibly a large number of vehicles before/after a concert or sporting event). These rules need to be effective as they impact a large number of vehicles. However in the case of semi-autonomous vehicles, this allows for a transportation system wide communication between all entities, thereby enabling a central transportation authority to dynamically regulate traffic as per the available information or traffic policies. Alternatively an intelligent system may be placed to constantly monitor traffic, anticipate traffic conditions and make traffic regulating decisions, which may be communicated to the vehicles for them to follow.

Traffic simulation ${ }^{17,18}$ allows the study of various ways or rules by which traffic can be regulated. Traffic simulation systems are classified into microscopic systems, macroscopic systems and mesoscopic systems. ${ }^{19}$ Our approach towards this domain is microscopic in nature, where we consider individual vehicle behaviour, when planned amongst a group of vehicles in a scenario. A commonly used traffic simulator is MITISM (Microscopic Traffic Simulator) ${ }^{20}$ where the surveillance system, traffic management, control and routing devices form the four main modules of simulation. Other common models include NETSIM,${ }^{21}$ INTEGRATION, ${ }^{22}$ DYNASMART ${ }^{23}$. All these simulators exhibit behaviours including lane changing, traffic merging, diversions, traffic lights, blockages, incidents, etc.

This work is motivated by related research by the authors, ${ }^{24,25}$ which studied the problem of trajectory generation for autonomous vehicles in a traffic environment with diverse speed capability vehicles. The problem was studied for unorganized traffic (where vehicles can move anywhere on the road and it is not necessary to drive in lanes) which was motivated from unorganized traffic systems presently witnessed and a theoretical analysis showing better efficiency for unorganized systems in diverse traffic. The study was however restricted to a straight road. It was observed that diversity leads to interesting driving behaviours. It is hence encouraging to study the effect of diverse unorganized traffic on the overall transportation system. This requires the need for a traffic simulator working with unorganized traffic and diverse vehicles. Creating a complete simulation system for unorganized traffic requires solving complicated sub-problems, and is outside the scope of this work. Here we report on a simulation system and initial results for diverse and organized traffic. Unfortunately none of the current simulation systems previously mentioned can be used, as they do not allow the integration of vehicle speed diversity as a factor.

The simulation system described here was built by taking a futuristic view where most of the vehicles would be semi-autonomous within an intelligent transportation infrastructure. A semi-autonomous 
vehicle may be defined as one that can be networked, with the ability to take basic driving instructions which may be implemented by a human driver or an autonomous vehicle itself. The aim is to exploit all possibilities with such traffic by closely observing each and every transportation entity. This notion opens a pool of new possibilities and issues, some of which are presented in the paper. Whilst vehicles being semiautonomous may not necessarily be a requirement in all cases, it will certainly benefit the system in making dynamic and fast changes, which cannot be done in the present traffic system.

With this simulation, our approach was to introduce a number of concepts in the present traffic system and to study the behaviour of vehicles under these concepts by a simulation. By this we could measure the effectiveness of the introduced concepts against the rules presently in the system for a diverse vehicle scenario. At the same time the attempt was to make a traffic simulation system that accounts for the ability to statically (at the start) or dynamically specify the applicability of all these concepts on various roads. The simulator also provides a base for our current and future research to make traffic systems more sophisticated, with the presented assumptions.

In this simulator we assume that the task is to enable a large number of vehicles to reach their destination from their source. The road network map is already available within the system. Each vehicle starts from its own source and attempts to reach its destination in the shortest time possible, in cooperation with the other vehicles. The vehicles emerge from their source at a predefined time, and leave the map on reaching their destination. At any time during the simulation process, the position of all the vehicles is assumed to be known. Further the vehicles can communicate with the central information system, which helps them in decision making.

The key contributions of the work are:

- The approach presents an integrated study of an intelligent transportation system covering all the various concepts which are separately studied in the literature.

- The study proposes an architecture for transportation systems of the future covering both decentralized vehicle control and a centralized management control.
- The approach is designed for diverse semiautonomous vehicles operating in a scalable environment, which is the likely future of transportation systems.

- The approach is a positive step towards the creation of a traffic simulation tool for diverse and unorganized traffic.

Traffic lights are an important aspect of a traffic management system. They ensure that vehicles reach their destinations on time, at the same time avoiding congestion. Both the order and duration of operation are important. The manner of handling traffic lights is discussed in section 2. Similarly speed lanes play a major part in the distribution of traffic. This especially becomes important in the case of vehicles with diverse speed capabilities. Deciding the speed limits for individual lanes is important, which in our system is discussed in section 3. Route planning deals with deciding on the roads to use to reach the destination. Continuous re-planning enables escaping from densely crowded roads, traffic regularization and the avoidance of traffic jams. Section 4 presents the route planning algorithm used in this system. Increased traffic density, slow traffic and a wide diversity in speed capabilities, especially at some times of the day and for some roads, necessitate the need to use roads as a reserved infrastructure. In section 5 we discuss the mechanism by which a road or a lane may be made available only by means of making a reservation. This enables important vehicles to reach their destination as early as possible, which includes emergency vehicles. Section 6 presents the general architecture of the system. Simulation results are given in Section 7 and concluding remarks are given in section 8 .

\section{Traffic Light Systems}

\subsection{Concept}

The waiting time for traffic lights to turn green may constitute a significant proportion of the time of journey. Efficient operation of traffic lights can lead to overall traffic efficiency. Presently employed traffic light systems allow traffic going from multiple sources to travel within a specified time. Usually this time is pre-specified to a threshold value. Common problems include having to wait for one's turn to cross when there is no other vehicle from the other direction, an equal waiting time for traffic on high density roads and low 
density roads, fixed traffic light operation times during the day, non-adaptability to changing traffic trends, having to wait for too many traffic light changes whilst travelling on a high traffic density road, etc. It is evident that intelligent traffic lights operating within an intelligent transportation system are capable of overcoming these problems.

The proposed traffic lights system considers both the number of vehicles as well as the operation time. It is assumed that the number of vehicles waiting to cross at each crossing is known in advance. In the case of networked semi-autonomous vehicles this task is trivial as the position of the vehicles is reasonably well known by GPS or a local mapping algorithm. Additionally sensors at the crossing region may help record the same data. Assume a crossing $c$ with intersecting roads $R=$ $\left\{R_{l}, R_{2}, R_{3}, R_{4}, \ldots R_{n}\right\}$. Vehicle information is noted in a data structure $Q_{c}$, which maintains a queue of all vehicles waiting at the crossing region. An entry comprises of the triplet $\left\langle V_{i}, R_{i}, t_{i}>\right.$ denoting the vehicle $V_{i}$ entered the crossing scenario (when it is ready to go over the crossing) at time $t_{i}$ from $\operatorname{road} R_{i} \in R$. The data structure is updated for every vehicle $\left(V_{i}\right)$ entering (1) or leaving (2) the crossing scenario.

$$
\begin{gathered}
Q_{c}=Q_{c} \cup\left\langle V_{i}, R_{a}, t_{i}\right\rangle \\
Q_{c}=Q_{c}-Q_{c}\left[V_{i}\right]
\end{gathered}
$$

Where $Q_{c}\left[V_{i}\right]$ is the entry of $V_{i}$ in $Q_{c}$.

The traffic lights operate in a manner such that at any time only one road has the green signal, while the signal for all other roads is red. All vehicles on the road in possession of the green signal can travel, irrespective of their exits. Lateral time is employed to clear the crossing region in-between light changes. The objective of the traffic lights is to reduce the maximum waiting time for any vehicle. Hence the order of traffic light changes is such that each change allows all traffic from road $R_{a}$ in possession of the longest waiting vehicle at crossing $c$ to cross. Let $D_{c}$ denote the road with the green traffic signal, which changes as per (3).

$$
D_{c}=R_{i}, i=\arg \min _{i,\left\langle V_{i}, R_{i}, t_{i}\right\rangle \in Q_{c}} t_{i}
$$

Traffic lights change as per the stated order if a traffic light change event (say $E_{c}$ ) occurs. After every change the traffic lights stay in the same mode until a maximum of $\eta$ vehicles have crossed or for a maximum of $T$ units of time. Here $\eta$ is taken as the minimum of a threshold value $\left(\eta_{t h}\right)$ and the present queue size $\left(\left|Q_{c}\left[D_{c}\right]\right|\right)$ for vehicles originating from the road which is currently green $\left(D_{c}\right) . T$ is the time threshold which is a constant. If there are no vehicles left on the road which is currently green, the traffic lights change. The change may however only occur if currently there is no other vehicle in the crossing area. Leaving this lateral time ensures that there is no deadlock in the crossing region. The pre-requisite for a change to occur is given by(4).

$$
E_{c}=T_{c} \geq T \vee \eta_{c}>\eta \vee\left|Q_{c}\left[D_{c}\right]\right|=\phi
$$

Here $T_{c}$ is the time elapsed since the last change and $\eta_{c}$ is the number of vehicles that passed since the last change.

Limiting both the maximum time and the maximum number of vehicles enables much better control of traffic when it contains diverse vehicles. Since the vehicles are semi-autonomous, this is realizable. An important criterion here is to keep $\eta_{c}$ as the minimum of the current queue size and a threshold. The effect of this is to discourage a newly entered vehicle from passing over the crossing region without waiting. This is because such a crossing might be at the cost of another waiting vehicle. If there are no other waiting vehicles, any change would automatically be in favour of the newly entered vehicle, which would be allowed to move. This is another way by which the heuristic of minimizing the waiting time can be realized.

\subsection{Simulations}

The purpose behind the simulations was to test the working of the proposed system, under diverse traffic conditions. A map was employed with a crossing at the centre. A random number of vehicles were generated, each with its own speed capability, and these vehicles were made to travel from one road to the other over the crossing. The emergence time of the vehicles was randomly fixed. This system of traffic light operation was compared to a system in which the lights were changed at regular intervals of $T$ units of time, which is the method frequently encountered in practice. In such a strategy a lot of time could be wasted if the light is green yet there are no vehicles which need to cross. The simulation system generated vehicles within random small time intervals that are uniformly distributed.

Another simpler traffic light change system was also studied. The change condition was kept largely as in (4), with the only difference being that changes were 
produced in a cyclic order rather than that proposed in (3). In all the simulations the average time of travel was used as a metric. These three algorithms were simulated for varying numbers of vehicles. A smaller number of vehicles in a scenario meant less densely occupied roads and vice versa. The resultant graph between the number of vehicles in the scenario and the average time of travel is shown in Figure 1(a). Every point on the graph represents a scenario with random entry time, exit time and speed. Hence the randomness has been smoothened by plotting a trend line produced by the moving average method. The simulation used arbitrary units of distance and time which are specific to the simulation tool and can relate to real world units proportionately.
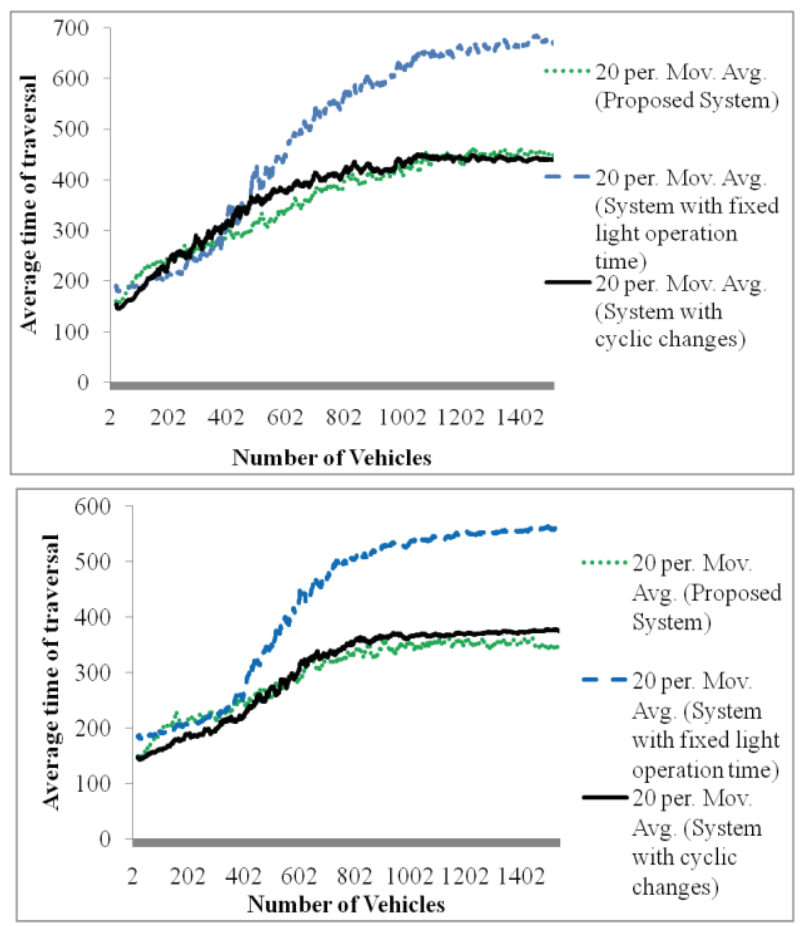

Fig. 1.Comparative analysis of traffic lights system for different number of vehicles (a) Traffic from all sides (b) Traffic from one side blocked.

The general trend in all the curves was an increase in the average time of traversal with increasing numbers of vehicles, until this time became a constant. The increase was due to increasing traffic density. On reaching a congested (saturated) traffic density any subsequent increase in the number of vehicles had no effect. The curve with fixed traffic light operation time was though much more inefficient when compared to the other two approaches which showed almost the same trend. That said, for middle density traffic the proposed system did exceed the system with cyclic crossing changes in terms of average traversal time. Small changes in average traversal time could though be regarded as insignificant, considering the random nature of the generation of vehicles. The significance of this was further highlighted by disallowing traffic generation from one side of the road, while the traffic lights operated in the same order. The resulting graph is shown in Figure 1(b). It is clear that the difference between the approaches is magnified, which shows the clear limitation of having fixed traffic light operation times.

There are two important parameters in the approach that govern the algorithmic performance. These are the time threshold $(T)$ and the threshold number of vehicles to cross $\left(\eta_{t h}\right)$. Here we analyze both these parameters. In the first experiment $T$ was varied and the average traversal time for the different situations was compared. The factor $\eta_{t h}$ was set to infinity, so that it had no effect on algorithmic performance. The study was broken down into a densely packed road (with 2000 vehicles) and a lightly packed road (with 250 vehicles). The resulting graph produced is shown in Figure 2(a) for a densely packed road and Figure 2(b) for a lightly packed road.
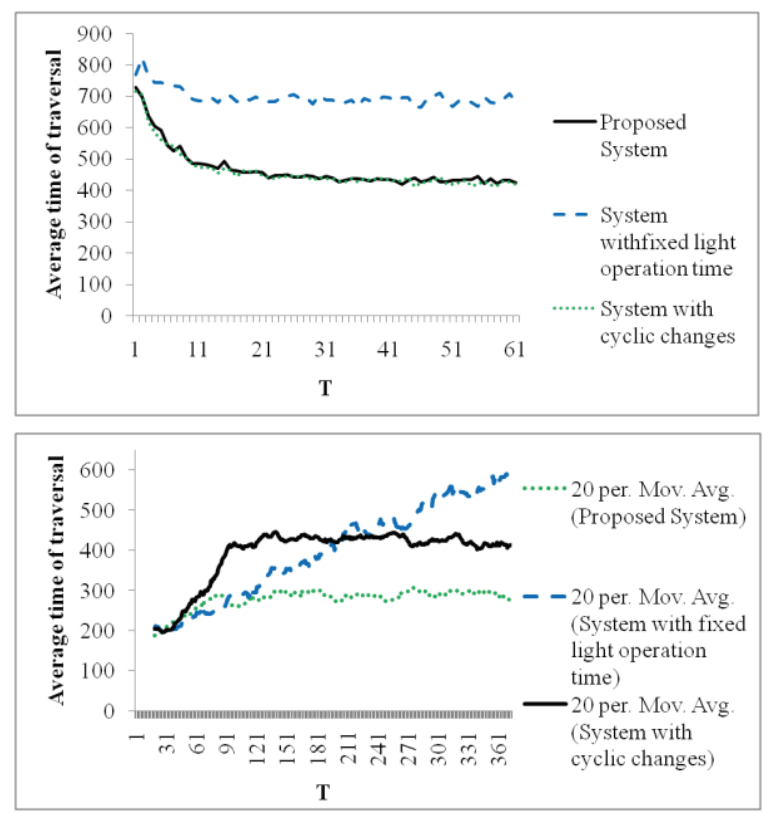

Fig. 2.Comparative analysis of traffic lights system for different values of $T$ (a) Densely occupied scenario (b) Lightly occupied scenario. 
Figure 2(a) shows that the general trend was an initial decrease in the average traversal time, as the time threshold was increased, which then became a steady value. Increasing this factor causes fewer traffic light changes, which as a result reduces the lateral switching time. The decrease reduces further as a very small proportion of vehicles are affected by the lateral time and the effect is balanced by the gain in reduced traversal time of the vehicles on the other roads at the crossing. This certainly suggests that making relatively few changes in traffic light signals could actually be beneficial overall. In a practical sense it would be sensible for no vehicle to stop at a crossing for more than two traffic light changes, which is practically observed mainly due to lower traffic density. However it may be noted that too few changes might increase the wait time for some vehicles drastically, while lowering the time for some other vehicles. Hence someone may be lucky enough to queue when the lights were about to change to green whereas another person might arrive at the front of the queue when the traffic lights have just changed to red. Maximum wait time is not a factor studied here, but in reality may be a value for a parameter setting.

The traversal time for fixed light operations is much higher than the other two cases, which seem to nearly follow each other. The entire scenario has start and end stages where the density is fairly low and a central stage of high density of vehicles. In the start and end stages the fixed light operation time algorithm performs very poorly as compared to the other two. Spending excessive time on a road with no vehicle in the queue is the major reason for this. Further in the central stage the other two approaches put stress on allowing the motion of vehicles which were in the queue at the time of a change (a rule used in light operation), whereas the fixed light operation time algorithm allows motion of any newly entrant vehicles as well. This decreases its effectiveness.

The same experiments performed with a lightly packed scenario show a different trend. The resulting graph is shown in Figure 2(b). The fixed light change algorithm showed a general increase in traversal time in line with increasing $T$. This was because of the waiting time when the queue was empty or was predominantly occupied by vehicles that had just arrived into the scenario and the traffic light was not changed. This waiting time increased with an increase in $T$ making the algorithm consistently inefficient. The other two systems however showed similar trends, with the proposed system being better as the value of $T$ was increased. The increase of this factor in this case had the effect of increasing the average traversal time, which soon settled around the same value. The increase was due to the fact that the road was lightly occupied and the increased time meant fewer changes which, as a result, increased the waiting time for vehicles. After some increase, the changes were caused only due to the vehicles in the queue being cleared rather than the time threshold. Hence this factor, on further increase, was not used. It may be observed that increasing $T$ increases the wait time for vehicles in a queue (un-preferred) but at the same time decreases the overheads of excessive crossing changes (preferred).

The other parameter of study was $\eta_{t h}$. It is natural that this factor plays no role in the system with fixed light operating times, and hence this study did not consider that system. This factor is discussed here separately for low and high density roads. For high density roads, there was a little decrease in traversal time on initial increase of this factor, but this soon became constant. The increase of this factor leads to fewer traffic light changes and hence less overhead. The corresponding graph is plotted in Figure 3. The irregular trends are because the actual points, rather than a trend line, are plotted. Experiments with low density roads showed that that the vehicles continued to travel without unnecessary queues. Since traffic lights did not wait in the case that the initial queue had cleared, the simulation continued. Hence this parameter had no effect on the performance and showed constant results for any value of set parameter.

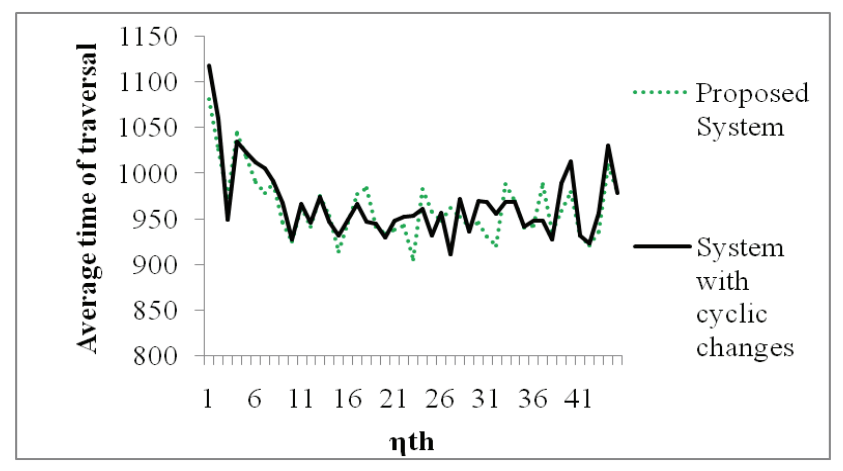

Fig. 3.Comparative analysis of traffic lights system for different values of $\eta_{t h}$ 


\section{Speed Lanes}

The idea of assigning different speed limits to different lanes is an important concept that especially comes into play if the traffic on a road has a high diversity in vehicles ranging from those with low speeds to those with high speeds. Having slower vehicles migrate onto all lanes clearly would make the traffic slow on all lanes, which is not good for higher preferential speed vehicles. Similar to the case of fixed traffic light operational times, the optimality of fixed speed limits for different speed lanes is questionable. The intention here therefore is to dynamically adjust the speeds limits of the various lanes. The central system vigilantly changes the speed limits depending upon the speed capability of the set of vehicles on the road, as they arrive and exit. The optimal speed limits of the lanes at any time may be a complex function depending upon the preferential speed distribution of the road. We however simplify the concept to some extent by assuming a uniform distribution. Let $\mathrm{V}=\left\{V_{i}\right\}$ be the set of vehicles on a road $R_{a}$. Let $s_{i}$ denote the preferential speed of a vehicle $V_{i}$. Let $\min _{i}\left(s_{i}\right)$ and $\max _{i}\left(s_{i}\right)$ respectively be the minimum and maximum preferential speeds exhibited by any vehicle on the road. Let the road have $b$ speed lanes. We can allocate the speed limits of the various speed zones (in increasing order of limits, measured from the left to right) by (5).

$$
L_{j}=\left(1-w_{j}\right) \min _{i}\left(s_{i}\right)+w_{j} \max _{i}\left(s_{i}\right)
$$

Where $w_{1}<w_{2}<w_{3} . .<w_{b}$

$w_{1}=0,0<w_{2}, w_{3}, \ldots w_{b} \leq 1$

It is important to realise that unlike the current traffic system, here the speed limit implies a lower bound, which is the least preferential speed that a vehicle must possess in order to drive in a particular lane. The upper bound is however set as infinity for every lane. Semi-autonomous vehicles may have their preferred speed set as per their capabilities as it would not be appropriate to force them drive subject to lower limits. This concept by itself results in overtaking capabilities for a vehicle with a reasonably high preferred speed. A vehicle on seeing another vehicle moving with a lower speed may opt to change its speed lane and drift rightward (the driving rule is assumed be on the left, which means overtaking on the right is preferred). After some time the overtaking vehicle would be ahead of the vehicle being overtaken. At this time if the vehicle finds another slower vehicle in front of it and there is no higher speed lane available, it can always drift leftward and return to its original speed lane. This is because the upper speed limit bound was set to infinity and it is quite valid for a high speed vehicle to drive on a speed lane with a lower bound. This would then complete the overtaking procedure. It is evident though that driving with a higher speed in a low speed lane may not be optimal, and hence eventually the vehicle would seek a chance to drift to the higher speed lane.

The system was studied via simulations. The map given was a simple straight road on which different vehicles were generated at different times with their own speeds. The road had 2 lanes (for each side of traffic - outbound and inbound). This made the lower bounds of the speed limits of the two lanes as 0 and (1w). $\min _{i}\left(s_{i}\right)+w \cdot \max _{i}\left(\mathrm{~s}_{\mathrm{i}}\right)$ by (5). We first attempted to study the behaviour of the parameter $w$. The study was performed separately under low density and high density conditions. The system was compared to a system that had no speed lanes and hence any vehicle could drive on any lane (even though the parameter $w$ plays no role for a system without speed lanes).

The graph produced on a densely packed road (with 2000 vehicles in the scenario) is shown in Figure 4(a). As the factor $w$ was increased so the speed limit for the high speed lane increased. As a result fewer vehicles were allowed to move to it. While this decreased the time of travel for the vehicles using the high speed lane, at the same time it led to the high speed lane being underutilized. As a result whenever the speed limit was increased, the decrease in travel time for the vehicles in the high speed lane was averaged out by an increase in travel time for the vehicles in the low speed lane. Conversely keeping this factor close to 0 makes the system equivalent to one with no speed lanes.

The same patterns may be observed in Figure 4(a). Lightly packed roads ideally do not require speed lanes as the various vehicles can easily pass each other without resulting in obstruction. In such a scenario high speed vehicles lose out as they have to spend time overtaking slower vehicles. However this lost time is very small in comparison with the time that the slower vehicles gain by having access to the high speed lane which increases the traffic bandwidth. The results of lightly packed road are shown in Figure 4(b). 

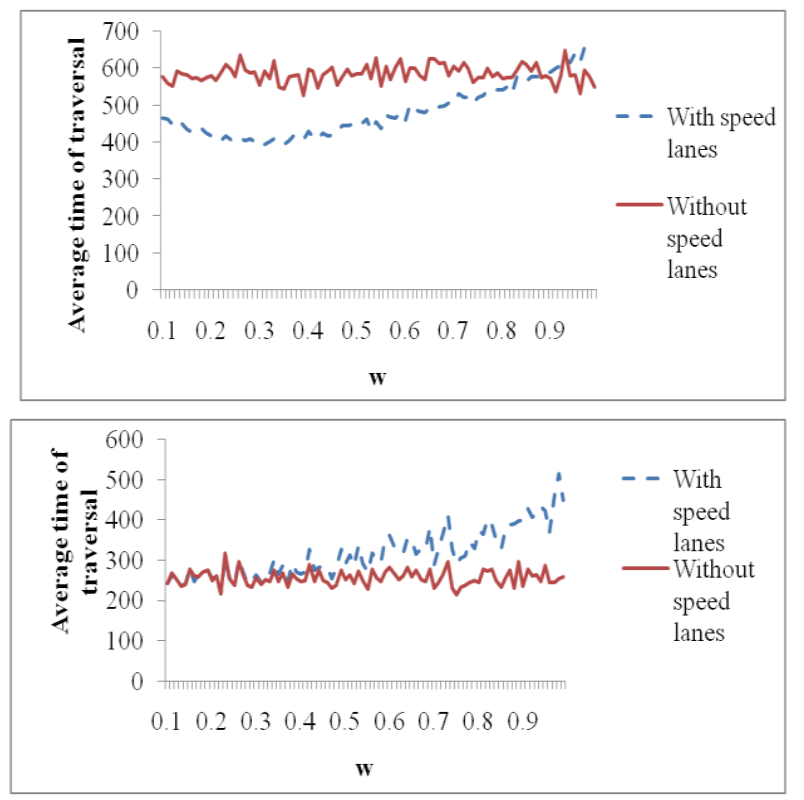

Fig. 4.Comparative analysis of speed lane system for different values of $w$ (a) Densely occupied scenario (b) Lightly occupied scenario.
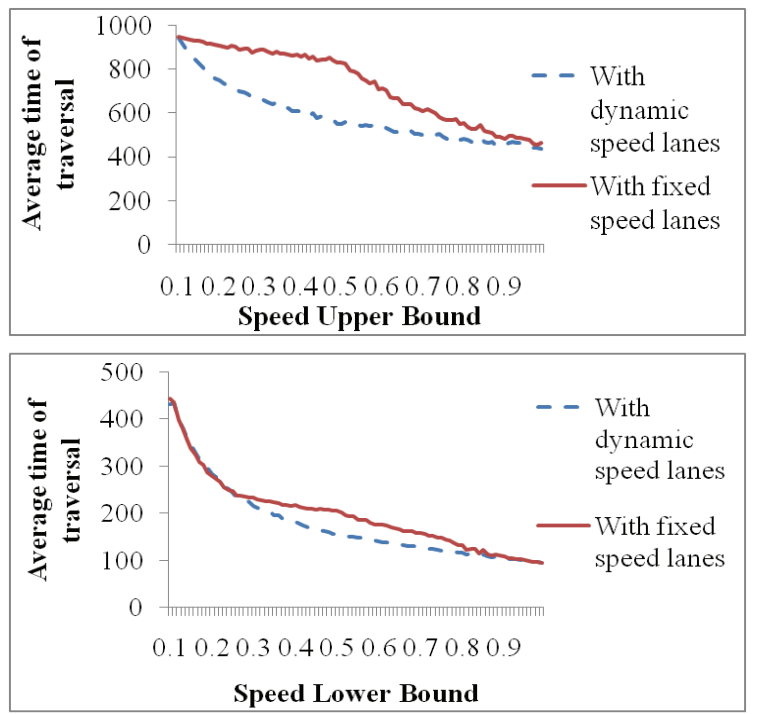

Fig. 5. Comparative analysis of speed lane system for different diversity of vehicles (a) Variable speed upper bound (b) Variable speed lower bound.

Diversity of traffic is a major factor studied in this paper, and it plays an important role in the speed lane system. In order to better test the system, we also studied the effect of a varying diversity of vehicle speeds on the algorithm performance. The simulation tool produced vehicles whose speeds lay within a specified upper and lower bound. We first experimented by changing the upper bound (at the same time keeping the lower bound fixed to a value of 0.2 unit distance per unit time, arbitrary units) and then subsequently experimented by changing the lower bound (keeping the upper bound fixed to a value of 1 unit distance per unit time, arbitrary units). The corresponding graphs are shown in Figure 5. Here we compared the time of traversal with that of a fixed speed lane system, where the speed limit (lower bound) of the high speed lane was fixed to a value of 0.5 unit distance per unit time. The general decrease in time in both cases is due to the increased average speed of the vehicle. It may be easily seen that the variable speed limit system nicely adapts the speed limits for enhanced performance.

\section{Route Planning}

Routing plays a major role in distributing traffic across the road network, enabling every vehicle to reach its destination in the shortest possible time in cooperation with the other vehicles. In reality it is common to have many vehicles using a popular road which enables quick access to a particular destination. This however leads to increased congestion and lower driving speeds for all, thereby resulting in reduced performance. Not considering other vehicles while planning one's own route may hence lead to poor results. The solution is to distribute traffic wisely on the roads, exploiting the entire transportation infrastructure for collective travel. An alternative longer road may be used, if it appears to have lesser traffic density as compared to the main road. However if the alternative road is too long, the choice may not be beneficial.

A Uniform Cost Search algorithm is used to route plan every vehicle in this approach. The aim of the Uniform Cost Search algorithm is to minimize the time of travel of all vehicles. Any road being selected for travelling is added a penalty which is proportional to the traffic density of the road. The central information system does know the current traffic density of the roads, but not the expected traffic density at the expected time of arrival of the vehicle on the roads. We hence predicted the traffic density using historical information of traffic flow. For roads near the current position of the vehicle, the current density is of a higher relevance, as it would not change much till the vehicle arrives at that road. However for roads far away from the current position of the vehicle, the prediction from 
historical data is more important as the current traffic scenario could change drastically. Hence the expected density at road $R_{a}$ at time $t$ may be given by (6).

$$
\rho\left(R_{a}, t\right)=\left\{\begin{array}{cc}
\rho_{\text {current }}\left(R_{a}\right) & t \leq \beta \\
\rho_{\text {historic }}\left(R_{a}, t\right) & t>\beta
\end{array}\right.
$$

Here $\rho_{\text {current }}\left(R_{a}\right)$ is the current traffic density and $\rho_{\text {historical }}\left(R_{a}, t\right)$ is the predicted traffic density. $\beta$ is the time until which the current traffic density is reliable. The total cost computed for a node $c_{l}$ when expanding from a node $c_{2}$ connected by a road $R_{a}$ for vehicle $V_{i}$ may hence be given by (7) and (8).

$$
\begin{gathered}
t\left(c_{1}\right)=t\left(c_{2}\right)+\frac{\left|R_{a}\right|}{s_{i}} \\
f\left(c_{1}\right)=f\left(c_{2}\right)+\alpha \cdot \rho\left(R_{a}, t\left(c_{1}\right)\right)
\end{gathered}
$$

Here $\alpha$ is the penalty constant, $t\left(c_{l}\right)$ is the time of arrival at $c_{l},\left|R_{a}\right|$ is the length of road $R_{a}$.

The route, as calculated by the Uniform Cost Search algorithm, is based on the current and predicted traffic, which changes with time. Vehicles may add in the desired route, vehicles may clear from the desired route, and irregular trends may make the actual traffic much different from the predicted traffic. The route needs to be constantly adapted against these changing trends. Continuous adaptation by all vehicles in the traffic scenario enables vehicles to collaboratively make an efficient travel plan. This adaptation is done by replanning the route. Once the vehicle is on a road, it is considered that it will not turn back, even if turning back leads to a better route. Hence maximum adaptation corresponds to re-planning on reaching every crossing. The re-planned route then reflects any changed traffic trends.

In order to test the working of this approach we generated a synthetic scenario with a straight road with source/destination at the extreme ends. We initially gave the road a very high traffic density. Two alternative roads of unequal length were however also available to be taken and these finally merged again with the main road. Vehicles were continuously generated on both sides of the road. The vehicles first drove along the straight road. After some time vehicles were seen on the smaller alternative, while some kept going on the straight road to maintain the density. Still later the larger alternative came into play and the vehicles used this for traversal as well.

An important factor of the algorithm is the parameter $\alpha$ which plays an important role in regulating traffic. We studied the effect of changing this factor on the system performance with a large number of vehicles. The algorithm was compared to a distance minimization Uniform Cost Search algorithm in which all the vehicles followed the same straight road and the other alternatives remained unused. The corresponding graph is given in Figure 6. Increasing this factor encouraged vehicles to balance traffic densities across the different road options. An initial decrease points to the vehicles preferring the alternative roads when the main road is dense, which is the right strategy to follow. However further increase of the factor encourages vehicles to take alternatives even though traffic on the main road is not very dense. This therefore increased the average time of traversal.

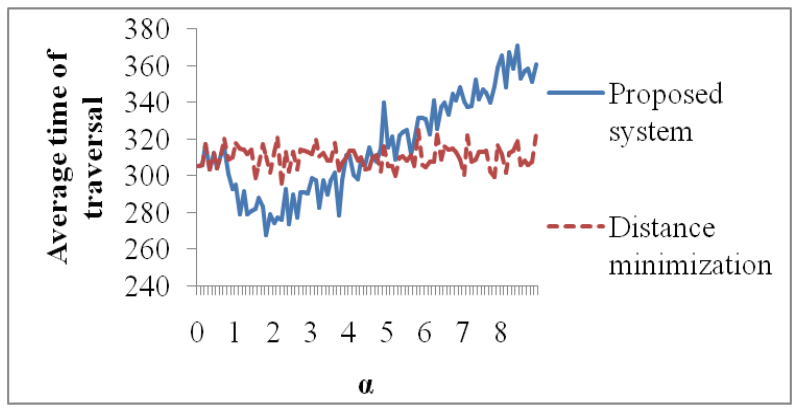

Fig. 6. Comparative analysis of routing system for different values of $\alpha$.

\section{Reservation}

Traffic systems may differentiate between important and non-important vehicles owing to their social importance. Roads may alternatively be seen as a business resource with possibilities of reserving a road or lane for periods of time exclusively or on shared basis. This is highly beneficial as reservation ensures decent travel speeds even though a general road may be heavily packed. This is especially important for roads where the density of traffic is usually high or is expected to be very high because of some event. In such a case it may be useful to have a road that can be reserved as per a pricing model. At the same time, other general traffic can still move on other available roads. An important factor here is the number of vehicles that may be reserved. Reserving too few vehicles would 
make the road underutilized. Reserving too many vehicles on the other hand could make the traffic on the reserved road slower than the traffic on the non-reserved roads, giving no incentive for reservation. Assuming a high density of overall traffic in the system, we further assumed that $p$ percent of these vehicles were reserved.

A map was generated for simulation that had a straight road from the source to destination and a rather long and highly curved alternative road. We made the straight road a reserved resource. Hence the reserved vehicles could travel straight on the road, while others would need to travel along the alternative, meandering, road. Unlike the map presented in section 4, the long length of the alternative road in this scenario made the use of the straight road to be highly beneficial. We varied the percentage of reserved vehicles $p$ and studied its effect on the average travel times of the reserved and the non-reserved vehicles. The graph is shown in Figure 7. Figure 7 also shows cases with all vehicles reserved, where they all used the straight road; and no vehicle reserved, in which they all used the diversion and the main road was left unused. The graph shows the increase in travelling time of the reserved vehicles as more and more of them travelled on the reserved road within the duration of simulation time. At the same time the average travel time for non-reserved vehicles decreases as their number is reduced. This may help in determining the number of vehicles to be reserved, keeping the trade-offs matched.

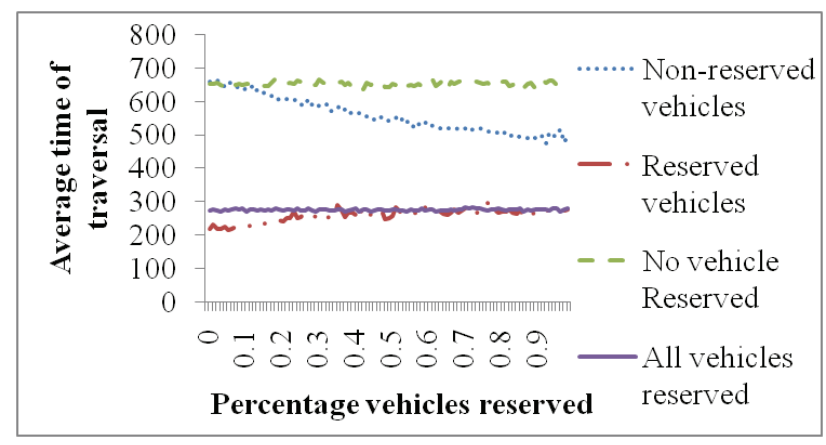

Fig. 7. Comparative analysis of road reservation system for different percentage of vehicles reserved.

A similar case may happen if only a lane on a road is made a reserved resource rather than a complete road. This makes it possible to use the existent road infrastructure for reservation as separate roads may not always be available giving reasonably alternate access. Reserved vehicles in such a case may be free to use general lanes and to overtake vehicles on the reserved lane but general vehicles may never be allowed to move to the reserved lanes. Another manner in which the problem can be seen is in the case of emergencies. It would be viable to reserve a lane for an emergency service vehicle rather than for the vehicle to wait for other vehicles to give way at the time of travel. The concepts of lane reservation are the same as road reservation. We again plotted a graph between the reserved percentage $p$ and the time of travel for reserved and non-reserved vehicles. All reserved and no reserved cases were also taken into account. The resultant graph is shown in Figure 8. Here there was an increase in the average time of traversal of vehicles as the number of vehicles reserved was increased. However it should be noted that in this case there was no difference between all vehicles reserved or no vehicles reserved. The graph shows the same trend.

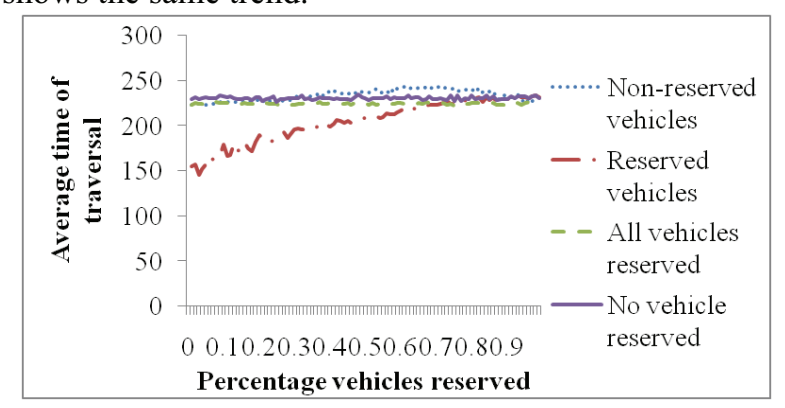

Fig. 8. Comparative analysis of lane reservation system for different percentage of vehicles reserved.

\section{General Architecture}

The general architecture of the simulator is given in Figure 9. The architecture clearly shows the four modules discussed separately. Each one of them links to the other for information passing. Central information is maintained by the central information system. The initial settings or scenario may be given to this system as an initial specification file. The central information system is queried by all the other modules for information. The general architecture may be separately studied for vehicle sub-system, reservation sub-system, traffic lights sub-system and speed lane sub-system.

The vehicle sub-system has a route planning algorithm that uses traffic information for deciding on the path. The lower level planner uses traffic information to decide the motion of the vehicles. This may be to follow the vehicle in front (or simply to drive straight ahead), to change lane, to stop at a crossing, to start from a 
crossing, or to turn at a crossing. Updated positions are always monitored and communicated to the central information system. For a lane change the vehicle must be informed of the speed limits of the lane. Additionally it must not be a reserved resource. Speed limits are always computed by the speed lane sub-system which gets all the traffic information from the central system for computation. The route planning sub-system must assess if the road is a reserved resource or not. The reservation sub-system gives directions to the lane subsystem and the vehicle's route planning module whether to use or not use the resource. Reservation may itself be separately handled by a system which is ultimately reflected in the central information system. The traffic lights sub-system operates the traffic lights based on the traffic behaviour on the various roads. The traffic light state is communicated to the vehicles on approaching a crossing.

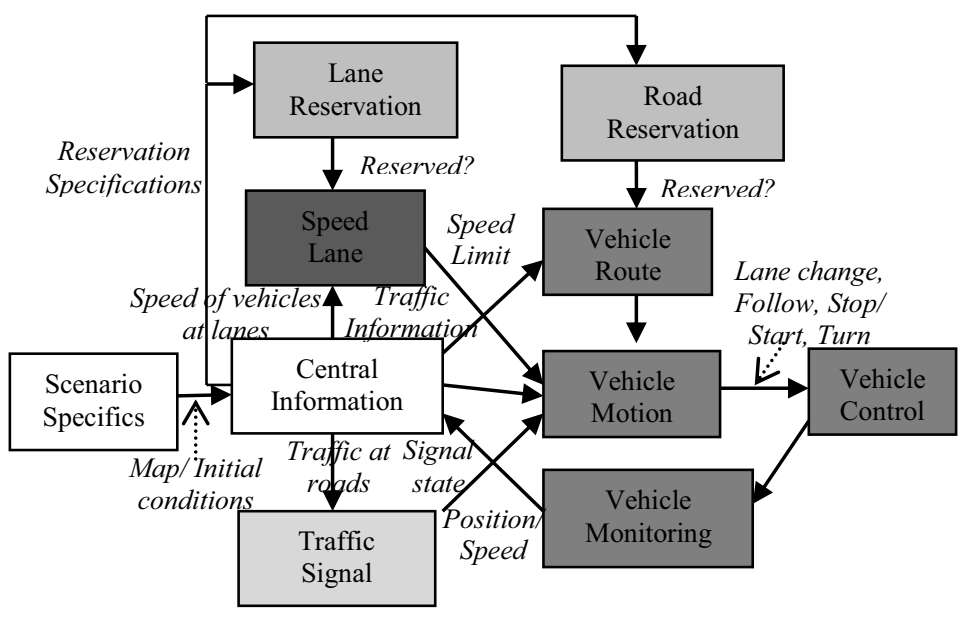

Fig. 9. Planning Architecture of the Simulator.

\section{Simulations}

So far we have proved separately the mechanism of working of intelligent traffic lights, speed lanes, route planning and road/lane reservation. All these concepts showcased benefits when compared to the current traffic management system. For traffic involving all these concepts at the same time it can hence be expected that the individual system gains would all contribute to overall transportation system performance. The traffic simulation system is aimed at running a large number of diverse vehicles using all the modules stated earlier. The testing methodology in this section is to invoke all the modules and test the performance in complicated traffic scenarios.
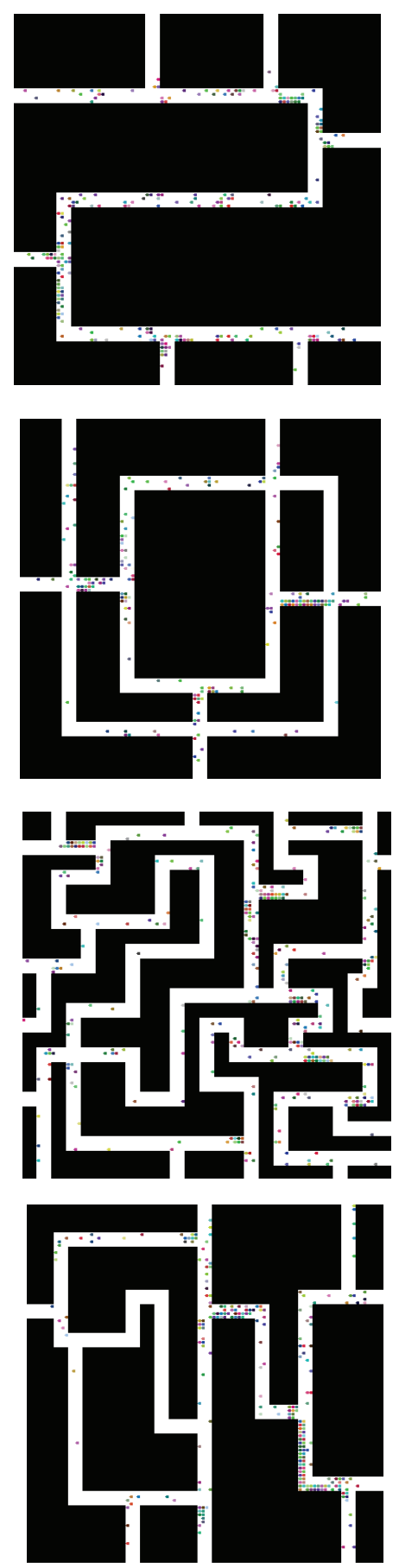

Fig. 10. Simulation Results. 
In order to make the testing easy, a utility is created which can take an image representation of the environment and parse it to produce the road network map. It then becomes easy to make maps and use them for testing. The only parameter to be given to the simulator is the demand or the number of vehicles generated per unit time. This is needed for control of the induced congestion which the algorithm tries to eliminate. The locations of the vehicles, initial speeds, emergence time, etc. are all set randomly. The initial and final positions are always kept at the extremity of the map so that the vehicles travel the maximum distance.

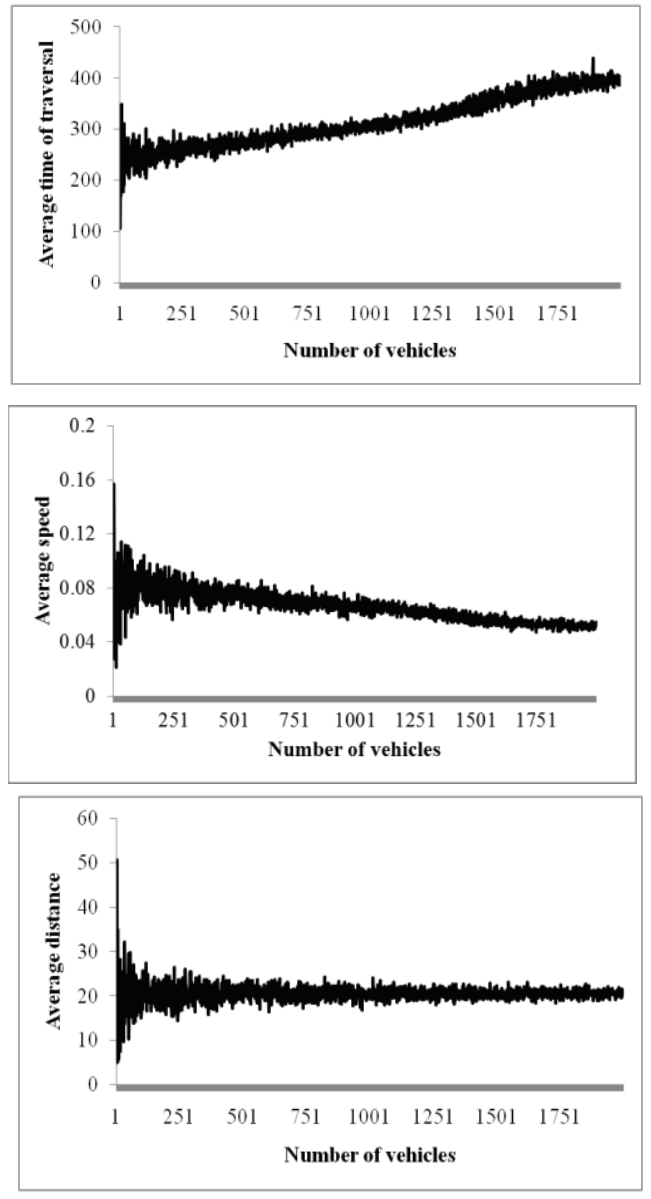

Fig. 11. Analysis for integrated scenario.

A number of maps were generated from the drawing utility tool and parsed to create a road network map. Large maps ensured that planning was a complex task. The densities were usually kept high as the benefits of the proposed system are particularly apparent in high density circumstances. To better assess the working of the algorithm, the vehicles were plotted on this map with different colors. Screenshots of some of the simulations for test scenarios are shown in Figure 10. For each simulation it was observed that the vehicles were able to easily reach their goals while avoiding congestions and excessive waiting times. In a congested setting with too many vehicles asked to go though some central regions, queues were formed for some time when a red light signal was apparent, but the queue cleared very quickly and the vehicles spread out to avoid congested areas.

The simulator measures a number of metrics which are indicators of the performance of the system. The primary performance indicator is the average travel time of the vehicles, which was the main metric used to assess the performance of the system. The other important metrics include the average travel distance and average travel speeds. To save space only one scenario is used for further analysis. The metrics for a different number of vehicles with all modules activated are shown in Figure 11. All units are arbitrary and specific to the simulation tool. The metrics can be mapped to real world units by multiplying by suitable constants.

Scenario specifications such as the sources and goals of vehicles were randomly generated. This randomness contributes to the oscillations seen in Figure 11. Based on a large number of simulations, it was observed that all the vehicles reached their destinations in acceptable times. Hence it can be ascertained that the simulation tool developed for the purpose was able to cooperatively plan the paths of the different vehicles involved.

\section{Conclusions}

Traffic density is constantly increasing and this puts a lot of stress on the present transportation infrastructure. Semi-autonomous vehicles with the option of communicating with other vehicles, road infrastructure and transportation management units are capable of efficiently planning themselves resulting in higher transportation efficiency. In this paper we have explored some of the various possibilities, where the aim was to build on the existing traffic management system by making each of its components intelligent and efficient. The proposed system was broken down into four main modules: traffic lights management, route planning, speed lane management and reservation. Each of these 
concepts resulted in better management of traffic, reducing the average traversal time of vehicles. The resulting system is a dynamically managed traffic system which attempts to make traffic flow as efficient as possible.

Diversity in vehicle speeds makes systems behave differently from general expectations. In this paper an attempt was made to investigate the effect of increased diversity of vehicular speeds on overall transportation performance. Slow vehicles can lead all traffic in a lane to be slow, resulting in reduced efficiency, and this has to be managed by the transportation system. In the simulations we saw that slow vehicles do affect the travel efficiency of the fast vehicles and the effect is unavoidable, however the effect is much lower in lower density traffic wherein fast vehicles have the option to overtake by lane changes.

Hence there is an advantage in eliminating high traffic density on roads, which was a key objective of the routing system. Alternatively, on high density roads, speed limits need to be intelligently adapted so as not to punish high speed vehicles too much by making them drive in low speed lanes while also not forcing vehicles to follow a slow vehicle ahead. Reservation signifies social diversity of vehicles. Expected dense traffic with diverse vehicles cannot be guaranteed a reasonable performance unless social diversity of vehicles is exploited as an additional factor.

Throughout this paper, we reported a traffic simulation system that works with a diverse set of vehicles, assuming the vehicles as semi-autonomous and in an intelligent transportation infrastructure. Initial results were given for different scenarios while experiments over simple policies were reported to showcase the working of major components of the transportation system. Our future research is aimed at extending the simulation framework for unorganized diverse traffic. Based on our prior experience it may be difficult to simulate a completely unorganized transportation system. However, working at a higher level of abstraction, an approximate system may be built. We intend to use this traffic simulation system as a base to design different algorithms and policies to enhance transportation performance.

It is important to study both human and vehicle driving models, neither of which were the core focus of the current research. Factors affecting microscopic behavior feasibility assessment, decision making and model based behavior implementation need to be modeled and studied. Further, human driving behaviors can change due to causes such as getting late, thereby leading to rushing, stress and fatigue, talking to someone, etc. These factors affect the way an individual drives and have implications on the overall traffic system. Such factors need to be critically examined.

The study will be specialized to real city maps and real traffic data (although a diversity of speeds may be assumed). Our first aim will be to focus on each subsystem separately, following which attempts will be made to design an overall system.

\section{Acknowledgements}

The authors wish to thank the Commonwealth Scholarship Commission in the United Kingdom and the British Council for their support of the first named author through the Commonwealth Scholarship and Fellowship Program (2010) - UK through award number INCS-2010-161.

\section{References}

1. Q. Ji, Z. Zhu, P. Lan, Real-time nonintrusive monitoring and prediction of driver fatigue. IEEE Trans. Veh. Technol. 53(4)(2004) 1052- 1068.

2. M. Althoff, O. Stursberg, M. Buss, Model-Based Probabilistic Collision Detection in Autonomous Driving. IEEE Trans. Intell. Transp. Syst. 10(2)(2009) 299-310.

3. G. K. Mitropoulos, I. S. Karanasiou, A. Hinsberger, F. Aguado-Agelet, H. Wieker, H. J. Hilt, S. Mammar, G. Noecker, Wireless Local Danger Warning: Cooperative Foresighted Driving Using Intervehicle Communication. IEEE Trans. Intell. Transp. Syst. 11(3)(2010) 539-553.

4. S. Cherng, C. Y. Fang, C. P. Chen, S. W. Chen, Critical Motion Detection of Nearby Moving Vehicles in a Vision-Based Driver-Assistance System. IEEE Trans. Intell. Transp. Syst. 10(1)(2009) 70-82.

5. L. Qingquan, Z. Zeng, B. Yang, Hierarchical model of road network for route planning in vehicle navigation systems. IEEE Intell. Transp. Syst. Magz. 1(2)(2009) 20 24.

6. X. Baro, S. Escalera, J. Vitria, O. Pujol, P. Radeva, Traffic Sign Recognition Using Evolutionary Adaboost Detection and Forest-ECOC Classification. IEEE Trans. Intell. Transp. Syst. 10(1)(2009) 113-126.

7. C. Urmson, C. Baker, J. M. Dolan, P. Rybski, B. Salesky, W. L. Whittaker, D. Ferguson, M. Darms, Autonomous Driving in Traffic: Boss and the Urban Challenge. AI Magz. 30(2)(2009) 17-29.

8. M. Montemerlo, J. Becker, S. Bhat, H. Dahlkamp, D. Dolgov, S. Ettinger, D. Haehnel, T. Hilden, G. Hoffmann, B. Huhnke, D. Johnston, S. Klumpp, D. 
Langer, A. Levandowski, J. Levinson, J. Marcil, D. Orenstein, J. Paefgen, I. Penny, A. Petrovskaya, M. Pflueger, G. Stanek, D. Stavens, A. Vogt, S. Thrun, Junior: The Stanford entry in the Urban Challenge. $J$. Field Rob. 25(9)(2008) 569-597.

9. J. Xu, G. Chen, M. Xie, Vision-guided automatic parking for smart car. In Proc. 2000 IEEE Symp. Intelligent Vehicles (2000), pp. 725-730.

10. J. E. Naranjo, C. Gonzalez, R. Garcia, R. de Pedro, LaneChange Fuzzy Control in Autonomous Vehicles for the Overtaking Maneuver. IEEE Trans. Intell. Transp. Syst. 9(3)(2008) 438-450.

11. G. V. Raffo, G. K. Gomes, J. E. Normey-Rico, C. R. Kelber, L. B. Becker, A Predictive Controller for Autonomous Vehicle Path Tracking. IEEE Trans. Intell. Transp. Syst. 10(1) (2009) 92-102.

12. I. Sen, D. W. Matolak, Vehicle-Vehicle Channel Models for the 5-GHz Band. IEEE Trans. Intell. Transp. Syst. 9(2)(2008) 235-245.

13. J. Huang, H. S. Tan, Error Analysis and Performance Evaluation of a Future-Trajectory-Based Cooperative Collision Warning System. IEEE Trans. Intell. Transp. Syst. 10(1)(2009) 175-180.

14. G. Rigas, P. Bougia, D. I. Fotiadis, C. D. Katsis, A. Koutlas, IWAY: Towards highway vehicle-2-vehicle communication and driver support, in Proc. IEEE Int. Conf. Systems, Man and Cybernetics(2008), pp. 33763381.

15. R. Kala, K. Warwick, Multi-Level Planning for SemiAutonomous Vehicles in Traffic Scenarios based on Separation Maximization. J. Intell. Rob. Syst 72(3-4)559590.

16. Y. Ma, M. Chowdhury, A. Sadek, M. Jeihani, Real-Time Highway Traffic Condition Assessment Framework Using Vehicle-Infrastructure Integration (VII) With Artificial Intelligence (AI). IEEE Trans. Intell. Transp. Syst. 10(4)(2009) 615-627.

17. M. Pursula, Simulation of traffic systems - an overview. J. Geogr. Inf. Des. Anal. 3(1)(1999) 1-8.

18. D. Helbing, Traffic and related self-driven many-particle systems. Rev. Mod. Phys. 73(4)(2001) 1067-1141.

19. D. Helbing, A. Hennecke, V. Shvetsov, M. Treiber, Micro-and macro-simulation of freeway traffic. Math. Comput. Model. 35(5-6)(2002) 517-547.

20. Q. Yang, H. N. Koutsopoulos, A Microscopic Traffic Simulator for evaluation of dynamic traffic management systems. Transp. Res. Part C: Emerg. Technol. 4(3)(1996) 113-129.

21. A. Byrne, A. de Laski, K. Courage, C. Wallace, Handbook of computer models for traffic operations analysis. Technical Report FHWA-TS-82-213 (Washington, D.C., 1982).

22. S. Algers, E. Bernauer, M. Boero, L. Breheret, C. D. Taranto, M. Dougherty, K. Fox, J. F. Gabard, Smartest project: Review of micro-simulation models. EU project No: RO-97-SC 1059 (1997).
23. R. Jayakrishnan, H. Mahamassani, T. Hu, An evaluation tool for advanced traffic information and management systems in urban networks. Transp. Res. Part C: Emerg. Technol. 2(3)(1994) 129-147.

24. R. Kala, K. Warwick, Planning Autonomous Vehicles in the Absence of Speed Lanes Using an Elastic Strip. IEEE Trans. Intell. Transp. Syst. 14(4)(2013)1743 - 1752.

25. R. Kala, K. Warwick,Motion Planning of Autonomous Vehicles in a Non-Autonomous Vehicle Environment without Speed Lanes. Engg. Appl. Artif. Intell. 26(56)(2013) 1588-1601. 